\title{
Markers of neutrophil extracellular traps predict adverse outcome in community- acquired pneumonia: secondary analysis of a randomised controlled trial
}

\author{
Fahim Ebrahimi (10 ${ }^{1}$, Stavros Giaglis ${ }^{2,3}$, Sinuhe Hahn ${ }^{3}$, Claudine A. Blum $^{1,4}$, \\ Christine Baumgartner ${ }^{5}$, Alexander Kutz ${ }^{4}$, Shane Vontelin van Breda ${ }^{2,3}$, \\ Beat Mueller ${ }^{4}$, Philipp Schuetz ${ }^{4}$, Mirjam Christ-Crain ${ }^{1,6}$ and Paul Hasler (1) ${ }^{2,6}$
}

Affiliations: ${ }^{1}$ Division of Endocrinology, Diabetes and Clinical Nutrition, Dept of Internal Medicine, University of Basel Hospital, Basel, Switzerland. ${ }^{2}$ Division of Rheumatology, Dept of Internal Medicine, Kantonsspital Aarau, Aarau, Switzerland. ${ }^{3}$ Dept of Biomedicine/University Women's Hospital, University of Basel Hospital, Basel, Switzerland. ${ }^{4}$ University Dept of Medicine, Kantonsspital Aarau, Aarau, Switzerland. ${ }^{5}$ Dept of General Internal Medicine, Inselspital, Bern University Hospital, Bern, Switzerland. ${ }^{6}$ These authors contributed equally to this work.

Correspondence: Paul Hasler, Division of Rheumatology, Medical University Dept, Kantonsspital Aarau, Tellstrasse, 5000 Aarau, Switzerland. E-mail: paul.hasleraksa.ch

@ERSpublications

Neutrophil extracellular traps predict higher risk for clinical instability and 30-day mortality in pneumonia http://ow.ly/Gxbh30iqbom

Cite this article as: Ebrahimi F, Giaglis S, Hahn S, et al. Markers of neutrophil extracellular traps predict adverse outcome in community-acquired pneumonia: secondary analysis of a randomised controlled trial. Eur Respir J 2018; 51: 1701389 [https://doi.org/10.1183/13993003.01389-2017].

ABSTRACT Neutrophil extracellular traps (NETs) are a hallmark of the immune response in inflammatory diseases. However, the role of NETs in community-acquired pneumonia (CAP) is unknown. This study aims to characterise the impact of NETs on clinical outcomes in pneumonia.

This is a secondary analysis of a randomised controlled, multicentre trial. Patients with CAP were randomly assigned to either $50 \mathrm{mg}$ prednisone or placebo for 7 days. The primary end-point was time to clinical stability; main secondary end-points were length of hospital stay and mortality.

In total, 310 patients were included in the analysis. Levels of cell-free nucleosomes as surrogate markers of NETosis were significantly increased at admission and declined over 7 days. NETs were significantly associated with reduced hazards of clinical stability and hospital discharge in multivariate adjusted analyses. Moreover, NETs were associated with a 3.8-fold increased adjusted odds ratio of 30-day mortality. Prednisone treatment modified circulatory NET levels and was associated with beneficial outcome.

CAP is accompanied by pronounced NET formation. Patients with elevated serum NET markers were at higher risk for clinical instability, prolonged length of hospital stay and 30-day all-cause mortality. NETs represent a novel marker for outcome and a possible target for adjunct treatments of pneumonia. 


\section{Introduction}

As a severe disease with high frequencies of morbidity and mortality, community-acquired pneumonia (CAP) ranks as one of the leading causes of hospital admission and is responsible for major socioeconomic costs [1]. Despite advances in antibiotic treatment and adjunct measures such as corticosteroids, hospitalised patients have persistently high mortality rates in the range of 5-15\% [2]. Limited respiratory function and pulmonary, cardiovascular or metabolic comorbidities increase the likelihood of a severe course of CAP and associated complications [3].

The immediate immune response to the inoculation, proliferation and spread of pathogenic microbes in the lower airways is primarily borne by polymorphonuclear neutrophil granulocytes (neutrophils), the most abundant leukocytes in humans. Once primed, they migrate from the blood vessels through the bronchial and alveolar interstitium to engage the offending organisms within the airspaces [4]. They also target microbes invading the pulmonary parenchyma or crossing into the bloodstream. The weapons at their disposal are the generation of reactive oxygen species, phagocytosis, release of granular enzymes and, as recently discovered, the extrusion of chromosomal DNA in reticular arrays termed neutrophil extracellular traps (NETs) [5]. In addition, they release chemokines and cytokines that recruit further cells of the innate and specific arms of the immune system, firing up inflammation that can be harmful and cause pulmonary dysfunction.

NETs consist of chromatin complexes of cell-free DNA (cfDNA) and histones, with attached neutrophil granular proteins (e.g. neutrophil elastase, myeloperoxidase (MPO) and cathepsin G), which trap and kill infectious microbes [5]. The process of NET formation entails a novel form of cell death that has been termed NETosis [6].

NETs have been extensively investigated in the laboratory and in inflammatory conditions such as pre-eclampsia, coagulation disorders, atherosclerosis and autoimmune diseases (e.g. rheumatoid arthritis, lupus erythematosus) $[7,8]$. However, clinical data on the role of NETs in infectious diseases are limited to case series in septicaemia. The characteristics of neutrophil activation to generate NETs in the initiation and resolution of pulmonary inflammation during the course of lower respiratory tract infections including CAP remain unknown.

Local and systemic effects of inflammation giving rise to measurable changes of mediators in the blood may be clinically useful for the guidance of decisions on therapy and for predicting the outcome of severe lower respiratory tract infections. Therefore, we aimed to explore the pattern and temporal dynamics of markers of NET formation and their associations with disease severity and relevant outcome measures of CAP. To investigate these questions, we chose a well-defined cohort of patients with CAP from a previous multicentre study, in which we recently showed that adjunct corticosteroids meaningfully shorten the time to clinical stability (TTCS) and length of hospital stay compared with placebo [9].

\section{Materials and methods}

\section{Study subjects and design}

This is a secondary analysis of an investigator-initiated, double-blind, randomised, placebo-controlled multicentre trial, in which adjunct treatment with prednisone in CAP was investigated. Details of the trial design and results have been published [9].

In brief, consecutive patients ( $\geqslant 18$ years) presenting with CAP were enrolled at emergency departments or medical wards of seven tertiary care hospitals in Switzerland within $24 \mathrm{~h}$ of presentation.

The conduct of the trial adhered to the Declaration of Helsinki and Good Clinical Practice guidelines, and ethical committees of all participating hospitals approved the study before patient recruitment. The trial was registered at ClinicalTrials.gov (identifier NCT00973154).

Support statement: This study was supported by grants from the Swiss National Science Foundation (SNSF PP0P3_123346) to M. Christ-Crain, and by the Nora van Meeuwen Häfliger Foundation and the Gottfried and Julia Bangerter-Rhyner Foundation. P. Hasler received support from the Research Council of the Kantonsspital Aarau. P. Schuetz was supported by the SNSF (PP00P3_150531/1). F. Ebrahimi was supported by Research Funds of the University of Basel, the Bangerter Foundation and the Swiss Academy of Medical Sciences (SAMS). Funding information for this article has been deposited with the Crossref Funder Registry.

Conflict of interest: F. Ebrahimi reports receiving grants from Research Funds of the University of Basel during the conduct of the study. S. Giaglis reports receiving grants from Kantonsspital Aarau Research Council during the conduct of the study and has a patent (US 20160061824 A1) pending. S. Hahn has a patent (US 20160061824 A1) pending. C.A. Blum reports receiving grants from Research Funds Endocrinology Kantonsspital Aarau during the conduct of the study. M. Christ-Crain reports receiving grants from the Swiss National Science Foundation, the Gottfried and Julia Bangerter-Rhyner Foundation and the Nora van Meeuwen Häfliger Foundation, during the conduct of the study. P. Hasler reports receiving grants from the Kantonsspital Aarau Research Council during the conduct of the study and has a patent (US $20160061824 \mathrm{~A} 1$ ) pending. 


\section{Methods}

Eligible patients were randomly assigned (1:1 ratio) to receive either $50 \mathrm{mg}$ prednisone or placebo daily for 7 days. Patients, treating physicians, investigators and data assessors were masked to treatment allocation.

Informed consent was obtained within $24 \mathrm{~h}$ of admission to hospital. All patients were treated according to international CAP consensus guidelines [10]. Baseline data included medical history, relevant comorbidities, clinical variables relating to pneumonia and all variables required for the calculation of the Pneumonia Severity Index (PSI) [11]. Blood samples obtained over the course of 7 days after admission were used to investigate the temporal dynamics of systemic NET surrogate markers. Clinical study data were gathered up to 30 days after admission. Structured follow-up telephone interviews for secondary outcomes after discharge were performed on day 30.

\section{Analysis}

The primary end-point was TTCS, defined as time to clinical stabilisation of vital signs at two consecutive measurements $\geqslant 12 \mathrm{~h}$ apart [9]. Secondary end-points included time to effective hospital discharge, all-cause mortality, duration of intravenous and overall antibiotic treatment, and CAP complications (including recurrence, acute respiratory distress syndrome, empyema, nosocomial infections until day 30, severe adverse events possibly related to CAP, intensive care unit (ICU) admission and readmission to hospital).

\section{Analysis of surrogates of NETS}

Blood samples (plasma and serum) from each patient were collected upon emergency department admission and on days 3,5 and 7, and processed as previously described [9].

Cell-free nucleosomes as surrogate of NETosis were measured in serum samples of the total study population using Human Cell Death Detection ELISA ${ }^{\text {PLUS }}$ (Roche Diagnostics, Basel, Switzerland). The concentrations of neutrophil elastase and MPO were measured in sera and plasma by sandwich ELISA, utilising the Elastase/ $\alpha 1$-PI complex ELISA kit (Calbiochem/EMD, Gibbstown, NJ, USA) and the human MPO ELISA kit (Hycult Biotech, Plymouth Meeting, PA, USA), respectively. To specifically characterise the source of cell-free nucleosomes as NET-associated MPO-DNA complexes, MPO-specific capture and subsequent DNA-specific detection antibodies were used as previously described [12]. For detailed methodology, see supplementary material [13-20].

\section{Statistical analysis}

Unless stated otherwise, categorical variables are expressed as number (percentage) and continuous variables as median (interquartile range (IQR)) or mean \pm standard deviation. In order to assess an integrated value of NET surrogates over 7 days, area under the concentration curve (AUC) values were calculated. For AUC calculations missing NETs, values were imputed using multiple imputation.

For temporal changes in biomarker levels, a multilevel mixed-effects linear regression model was applied. Associations of comorbidities with baseline levels of cell-free nucleosomes were analysed by univariate and multivariate linear regression models. For the primary end-point, we used a multivariate Cox proportional hazards model based on a binary outcome of achieving or not achieving clinical stability. For all secondary end-points, we calculated unadjusted and adjusted estimates of the effect size and corresponding 95\% confidence intervals using linear, logistic or Cox proportional hazards regression as appropriate. All multivariate models were adjusted for the same variables: treatment group, patient age, sex, body mass index, smoking status, PSI and comorbidities (congestive heart failure, chronic kidney disease, cerebrovascular disease, peripheral artery occlusive disease, chronic obstructive pulmonary disease (COPD), asthma), as well as clinical variables (days of symptoms before admission, systemic inflammatory response syndrome (SIRS) criteria, C-reactive protein and white blood cell count). Kaplan-Meier curves were used to illustrate TTCS based on NET quartiles (highest quartile versus lower three quartiles).

All statistical analyses were performed using Stata version 14.2 (StataCorp, College Station, TX, USA) and tests were done at a two-sided 5\% significance level with two-sided 95\% confidence intervals.

\section{Results}

\section{Patient characteristics}

Overall, 310 patients were randomly selected from the total study population of 785 patients for the current analysis. Baseline characteristics of treatment and placebo subgroups are presented in table 1. The median age was 75 years and $64.2 \%$ were male. The burden of pre-existing pulmonary disease was relatively low, with a positive smoking history in $23.2 \%$ and a history of COPD in $17.4 \%$. At hospital admission, the median duration of symptoms was 4 days and approximately one-fifth (21.9\%) of the patients had been pre-treated with an antibiotic. Overall, $78.5 \%$ of the patients had two or more SIRS 
TABLE 1 Baseline characteristics and clinical variables of enrolled patients



Data are presented as $n$, median (interquartile range) or $n$ (\%), unless otherwise stated. BMI: body mass index; COPD: chronic obstructive pulmonary disease; SIRS: systemic inflammatory response syndrome; PSI: Pneumonia Severity Index. \#: the PSI is a clinical prediction rule to calculate the probability of morbidity and mortality in patients with community-acquired pneumonia [11]; PSI risk class I corresponds to age $\leqslant 50$ years and no risk factors ( $\leqslant 50$ points), risk class II to $51-70$ points, risk class III to $71-90$ points, risk class IV to $91-130$ points and risk class $\mathrm{V}$ to $>130$ points.

criteria, and about half (53.6\%) had severe pneumonia classified in the high-risk PSI classes IV and V, respectively.

Temporal dynamics of NETs

At baseline, serum cell-free nucleosomes as surrogates of NET formation were highly increased $(2.67 \pm 1.32$ absorbance units $(\mathrm{AU})$ ) compared with values in healthy controls reported as $\sim 0.2 \mathrm{AU}[13,15,17,18]$. They subsided gradually until day 7 (day 3: $1.48 \pm 1.03 \mathrm{AU}$; $<<0.0005$; day 5: $1.19 \pm 0.87 \mathrm{AU}$; $\mathrm{p}<0.0005$; 


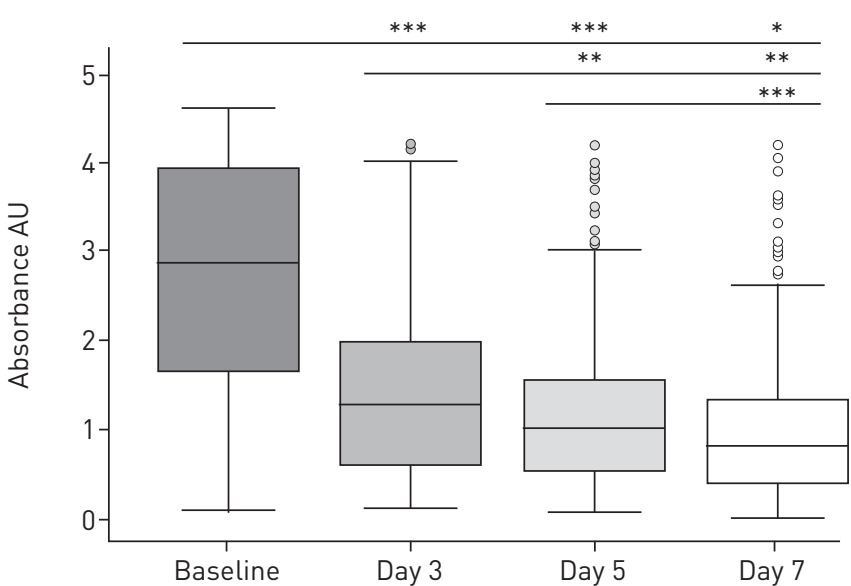

FIGURE 1 Box plots for levels of cell-free nucleosomes as a surrogate marker of neutrophil extracellular traps (NETs) in serum samples of the whole cohort $(n=310)$ at four different time-points (baseline, and days 3 , 5 and 7$)$ in the course of community-acquired pneumonia. AU: absorbance units $\left(A_{405 \mathrm{~nm}}-A_{490 \mathrm{~nm}}\right)$. Each box signifies the upper and lower quartiles, while the median is represented by a line within the box. Whiskers represent the upper and lower adjacent values; outliers are depicted as circles. At admission, mean levels of NETs were markedly increased $(2.67 \pm 1.32 \mathrm{AU}$ ) and decreased gradually over time (day 3: $1.48 \pm 1.03 \mathrm{AU}$; $p<0.0005$; day 5: 1.19 $\pm 0.87 \mathrm{AU} ; p<0.0005$; day 7: $0.98 \pm 0.79 \mathrm{AU} ; p=0.042$ ). Mixed-effects linear regression model. *: $p<0.05 ;{ }^{* *}: p<0.005 ;{ }^{* * *}: p<0.0005$.

day 7: $0.98 \pm 0.79 \mathrm{AU} ; \mathrm{p}=0.042$; figure 1 ), when they were still markedly elevated compared with values of randomly selected healthy controls (characteristics of healthy controls are shown in supplementary table S1). In the plasma, cell-free nucleosomes were much lower and maximum values were reached at day 3 (data not shown). Quantification of MPO-DNA complexes showed a high correlation with values of cell-free nucleosomes, indicating that they represent NETs $\left(r=0.55, R^{2}=0.3, p=0.012\right.$ ) (supplementary figure S3).

\section{Associations of initial NET surrogates with demographic characteristics, comorbidities and clinical variables}

Multivariate linear regression models were used to investigate predictors of increased markers of NETosis at baseline. In univariate analysis, advanced age was associated with decreased levels of NETs (data not shown), although this association was no longer significant after full adjustment for multiple covariates (table 2). Smokers with a greater past exposure to tobacco, as measured by pack-years, had significantly lower NET levels on hospital admission ( -0.05 AU per 10 pack-years; $p=0.042$ ). There was likewise an inverse association of NET levels with heart failure. Patients with a history of heart failure had significantly lower NET levels on hospital admission compared with patients without heart failure $(2.33 \pm 1.45$ versus 2.76 $\pm 1.28 \mathrm{AU} ; \mathrm{p}=0.036$ ). This association remained robust in a fully adjusted model. As NETs are generated by neutrophils, NET levels were correspondingly associated with elevations of white blood cell counts as well as neutrophil counts (table 2).

\section{Association between markers of NETs and disease outcome measures}

Relevant disease outcome measures were analysed for NET surrogates at baseline as well as for the AUCs, integrating values of NET surrogates over 7 days. The primary end-point, i.e. TTCS, was significantly longer in patients from the highest AUC NET quartile (median (IQR) 5.0 (2.6-9.0) days) compared with the lower three quartiles (4.0 (2.0-7.4) days) with an adjusted hazard ratio (HR) of 0.97 (95\% CI 0.940.99; $\mathrm{p}=0.041$; figure 2 and table 3); a HR<1.0 corresponding to prolonged TTCS. For high baseline NET levels, a trend towards prolonged TTCS was noted (adjusted HR 0.91, 95\% CI 0.82-1.01; p=0.088).

Median (IQR) time to effective discharge from hospital was longer in patients with the highest quartile of AUC NET levels (9.0 (5.0-14.0) days) compared with the three lower quartiles (7.0 (5.0-11.0) days) with an adjusted HR of 0.90 (95\% CI $0.82-0.99 ; \mathrm{p}=0.042$ ). In fully adjusted logistic regression analyses, increased NET levels at admission were associated with a 3.8-fold odds ratio of 30-day mortality. In addition, a stepwise increase in mortality was found with mounting quartiles of NETs.

Higher markers of NETs at baseline were associated with a 0.52 -day prolongation of i.v. antibiotic treatment (regression coefficient $0.52,95 \%$ CI $0.1-0.94 ; \mathrm{p}=0.015$ ). There was no association of NETs with a composite end-point of complications connected with CAP or the probability of ICU admission (table 3 ). 
TABLE 2 Association of baseline neutrophil extracellular trap (NET) levels with demographic characteristics, comorbidities and clinical variables

Adjusted (multivariate) linear regression coefficient $(95 \% \mathrm{Cl})$

p-value

\begin{tabular}{lcc}
\hline General characteristics & & \\
Age at study entry & $-0.001(-0.02-0.006)$ & 0.292 \\
Male & $0.118(-0.22-0.463)$ & 0.502 \\
BMI & $-0.001(-0.03-0.026)$ & 0.892 \\
Ever-smoker & $0.147(-0.23-0.529)$ & 0.450 \\
Pack-years (per 10 years) & $-0.05(-0.10--0.00)$ & 0.042 \\
Comorbidities & & \\
Diabetes mellitus & $0.160(-0.23-0.559)$ & 0.428 \\
Insulin treatment & $0.318(-0.25-0.895)$ & 0.279 \\
COPD & $-0.01(-0.42-0.395)$ & 0.943 \\
Asthma & $-0.24(-0.99-0.504)$ & 0.522 \\
Heart failure & $-0.46(-0.89--0.02)$ & 0.036 \\
Hypertension & $0.041(-0.28-0.370)$ & 0.802 \\
Cerebrovascular disease & $-0.23(-0.79-0.326)$ & 0.410 \\
Peripheral artery occlusive disease & $-0.26(-0.93-0.409)$ & 0.442 \\
Renal insufficiency & $-0.04(-0.38-0.290)$ & 0.775 \\
Neoplastic disease & $-0.36(-1.0-0.283)$ & 0.270 \\
Clinical variables & & \\
Steroid pre-treatment & $0.327(-0.41-1.07)$ & 0.385 \\
Antibiotic pre-treatment & $-0.02(-0.40-0.355)$ & 0.891 \\
Days with symptoms & $0.004(-0.01-0.021)$ & 0.648 \\
Temperature & $-0.01(-0.18-0.164)$ & 0.902 \\
Oxygen saturation & $0.006(-0.04-0.056)$ & 0.790 \\
PSI score & $0.001(-0.001-0.007)$ & 0.886 \\
Positive blood culture & $0.115(-0.38-0.615)$ & 0.649 \\
Pathogen detection & $-0.05(-0.41-0.312)$ & 0.782 \\
Neutrophil granulocytes & $0.033(0.006-0.061)$ & 0.016 \\
Leukocyte count & $0.019(-0.00-0.043)$ & 0.116 \\
\hline Baseline NET levels & & \\
\hline
\end{tabular}

Baseline NET levels were negatively associated with the quantity of pack-years and a history of heart failure, but positively associated with counts of neutrophils. $p<0.05$ statistically significant. BMI: body mass index; COPD: chronic obstructive pulmonary disease; PSI: Pneumonia Severity Index.

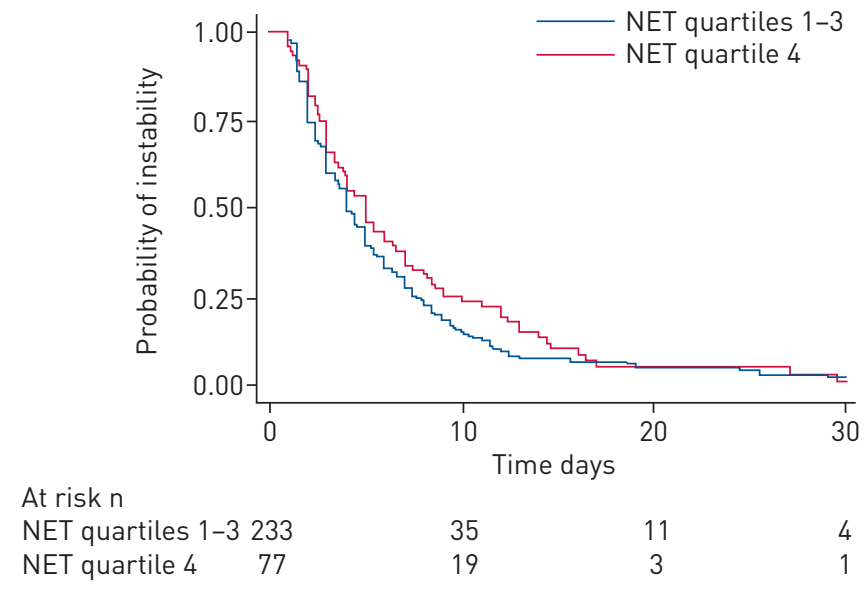

FIGURE 2 Kaplan-Meier curve of time to clinical stability (TTCS) for area under the concentration curves of neutrophil extracellular traps (NETs), an integrated value over 7 days: NET quartile 4 (highest quartile) versus NET quartiles 1-3. Higher NET levels are associated with prolonged TTCS ladjusted hazard ratio $0.97,95 \% \mathrm{Cl}$ $0.94-0.99 ; p=0.041)$. 
TABLE 3 Overview of primary and secondary end-points: neutrophil extracellular traps (NETs)

\begin{tabular}{|c|c|c|c|c|c|}
\hline \multirow{2}{*}{$\begin{array}{l}\text { Quartiles 1-3 } \\
\text { AUC NETs }\end{array}$} & \multirow{2}{*}{$\begin{array}{l}\text { Quartile } 4 \\
\text { AUC NETs }\end{array}$} & \multicolumn{2}{|l|}{ NETs at baseline } & \multicolumn{2}{|l|}{ AUC of NETs } \\
\hline & & $\begin{array}{l}\text { Multivariate adjusted } \\
\text { regression analysis: OR, } \\
\text { HR, coefficient }(95 \% \mathrm{CI})\end{array}$ & $\mathrm{p}$-value & $\begin{array}{l}\text { Multivariate adjusted } \\
\text { regression analysis: OR, } \\
\text { HR, coefficient }(95 \% \mathrm{CI})\end{array}$ & $p$-value \\
\hline
\end{tabular}

\begin{tabular}{|c|c|c|c|c|c|c|}
\hline \multicolumn{7}{|l|}{ Primary end-point } \\
\hline TTCS days & $4.0(2.0-7.4)$ & $5.0(2.6-9.0)$ & $0.91(0.82-1.01)^{+}$ & 0.088 & $0.97(0.94-0.99)^{+}$ & 0.041 \\
\hline \multicolumn{7}{|l|}{ Secondary end-points } \\
\hline $\begin{array}{l}\text { Time to effective } \\
\text { hospital discharge days }\end{array}$ & $7.0(5.0-11.0)$ & $9.0(5.0-14.0)$ & $0.90(0.82-0.99)^{+}$ & 0.042 & $0.96(0.94-0.99)^{+}$ & 0.012 \\
\hline Death (30 days) & $9(3.9)$ & $4(5.2)$ & $3.81(1.39-10.4)^{\S}$ & 0.009 & $1.27(1.05-1.54)^{\S}$ & 0.012 \\
\hline $\begin{array}{l}\text { Total duration of antibiotic } \\
\text { treatment days }\end{array}$ & $10.0(7.0-12.0)$ & $11.0(9.0-14.0)$ & $0.38(-0.01-0.84)^{f}$ & 0.099 & $0.07(-0.01-0.20)^{f}$ & 0.253 \\
\hline $\begin{array}{l}\text { Intravenous antibiotic } \\
\text { treatment days }\end{array}$ & $5.0(4.0-7.0)$ & $6.0(4.0-10.0)$ & $0.52(0.10-0.94)^{f}$ & 0.015 & $0.13(0.01-0.24)^{f}$ & 0.021 \\
\hline CAP complications $\# \#$ & $77(33.1)$ & $17(22.1)$ & $0.97(0.78-1.22)^{\S}$ & 0.853 & $0.99(0.93-1.06)^{\S}$ & 0.92 \\
\hline ICU admission & $21(9.0)$ & $6(7.8)$ & $0.90(0.58-1.39)^{\S}$ & 0.645 & $0.97(0.87-1.09)^{\S}$ & 0.707 \\
\hline
\end{tabular}

Data are presented as $\mathrm{n}$, median (interquartile range) or $\mathrm{n}(\%)$, unless otherwise stated, and adjusted for multiple variables (see Materials and methods). AUC: area under the concentration curve; TTCS: time to clinical stability; CAP: community-acquired pneumonia; ICU: intensive care unit. \#: $n=233 ;{ }^{~ ๆ}: n=77 ;{ }^{+}$: hazard ratio; ${ }^{\S}$ : odds ratio; ${ }^{f}$ : regression coefficient; ${ }^{\# \#}$ : CAP complications: recurrence, acute respiratory distress syndrome, empyema, nosocomial infections until day 30 , serious adverse events possibly related to CAP, ICU stay, readmission to hospital. $\mathrm{p}<0.05$ statistically significant.

\section{Effects of corticosteroids on NETS}

In accordance with the main results from the original cohort, randomised treatment with prednisone over 7 days was associated with a significantly shorter median (IQR) TTCS (3.4 (2.0-6.0) versus 5.4 (3.09.0) days) and length of hospital stay (7.0 (5.0-11.0) versus 8.0 (5.0-13.0) days).

Corticosteroid treatment correlated with a slower decrease in levels of NET surrogates over time (figure 3). While there was no significant difference in mean values of cell-free nucleosomes as NET markers up to 3 days, corticosteroid treatment correlated with significantly higher NET formation on day 5 compared with placebo $(1.09 \pm 0.88 \mathrm{AU}$ in placebo group versus $1.31 \pm 0.84 \mathrm{AU}$ in steroid group; $\mathrm{p}=0.023)$ and day 7 $(0.79 \pm 0.74$ versus $1.19 \pm 0.80 \mathrm{AU} ; \mathrm{p}<0.0005)$. However, overall AUC values were not influenced by adjunct corticosteroid treatment compared with placebo (11.27 \pm 4.7 versus 10.74 $\pm 5.1 \mathrm{AU} ; \mathrm{p}=0.267)$. Likewise, patients from the placebo group presenting stable or rising markers of NETs after day 3 had higher hazards of clinical stability (adjusted HR 1.75, 95\% CI 1.06-2.89; $\mathrm{p}=0.027$ ).

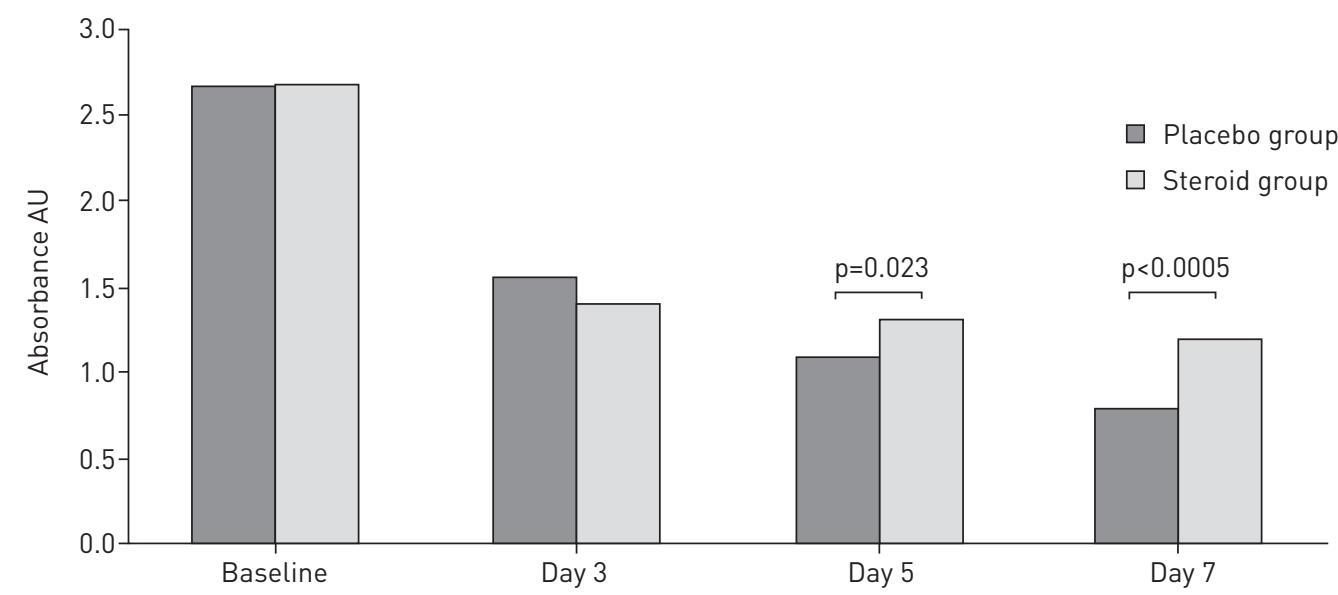

FIGURE 3 Mean absorbance of neutrophil extracellular traps (NETs): placebo group versus steroid group. AU: absorbance units $\left(A_{405 \mathrm{~nm}}-A_{490} \mathrm{~nm}\right)$. On day $5(1.09 \pm 0.88$ versus $1.31 \pm 0.84 \mathrm{AU}$, respectively; $\mathrm{p}=0.023)$ and day 7 $(0.79 \pm 0.74$ versus $1.19 \pm 0.80 \mathrm{AU}$, respectively; $p<0.0005)$ prednisone treatment was associated with significantly higher NET formation compared with placebo. 
With regard to the beneficial effects of prednisone on clinical outcomes, we noted a significant effect modification by NET markers on TTCS ( $p_{\text {interaction }}=0.008$ ). Patients with levels of NETs in the highest quartile shared a 1.8-fold higher adjusted HR of clinical stability upon prednisone treatment compared with patients in the three lower quartiles (adjusted HR 1.79, 95\% CI 1.03-3.12).

\section{Discussion}

The key findings of this study on NETs in patients with CAP are 3-fold. First, serum levels of cell-free nucleosomes as a surrogate marker for NETs are vastly increased in the context of CAP and remain at remarkably elevated levels even after 7 days. As the values for the ELISA of nucleosomes reflect a logarithmic scale, the actual magnitude of NET formation will be inclined to be underestimated, as it is in the range of mean elevations to the power of two to even four when compared with healthy volunteers $[13,15,17,18]$ and is considerably higher than that observed in autoimmune diseases such as rheumatoid arthritis with well-controlled activity [13]. While NETs are formed to fight infectious agents by entrapping and killing a wide variety of bacteria [21] and other microbes [22, 23], excessive activation of neutrophils and consecutive production of NETs are associated with the pathology of a number of airway disorders [24], such as COPD [25-27], impairment of alveolar gas exchange, lung dysfunction [28-31], and ultimately increased risk of early and late death.

Additionally, as measurements of NET surrogate markers were performed in serum samples, the results not only represent pre-formed systemic NETs, but also allow estimation of the propensity of pre-activated neutrophils to release NETs during coagulation. The magnitude of NETosis measured in the serum at baseline correlates with neutrophil and leukocyte cell count, but was reduced with the presence of heart failure. This observation may be due to pooling of neutrophils in the pulmonary circulation on account of reduced cardiac output [32]. While nicotine has been described to be a potent inducer of NETosis [33-35], in our cohort the number of pack-years was negatively associated with baseline levels of NETs, which could possibly be explained by exhaustion due to chronic activation of NETosis-related pathways.

The second important finding is that high levels of NETs at admission as well as a high integral of NETs during the course of CAP over 7 days (AUC) were strong independent risk factors for adverse outcome. Patients in the highest quartile had a median prolongation of TTCS by 1 day and of the length of hospital stay by 2 days, accompanied by increased duration of i.v. antibiotic treatment by 0.5 days. The differences in TTCS seemed to be less prominent in patients with very short ( $<5$ days) or prolonged ( $>15$ days) TTCS.

Remarkably, the results show that NET values at admission are associated with an almost 4 -fold odds of all-cause mortality in CAP patients, representing a novel biomarker for outcome prediction, i.e. for identification of patients at risk.

These findings are consistent with previous observational studies that examined the association between cell-free DNA or nucleosomes and the risk of mortality in ICU patients. In a prospective observational cohort study from Finland on 225 patients with severe sepsis, admission concentrations of cfDNA were higher in ICU nonsurvivors compared with survivors [36]. Likewise, a retrospective observational study on 80 severe sepsis patients revealed high prognostic utility of cfDNA levels to predict ICU and hospital mortality [37]. Further observational studies support evidence of NETosis as a hallmark in the pathogenesis of systemic inflammation, hypercoagulability and sepsis [38-40]. These observations are in line with experimental data showing that intense pulmonary NET generation determines the disease severity in both viral and bacterial pneumonia [22, 41]. Seeking evidence that measured cell-free nucleosomes effectively mirrored NETs, we quantified complexes of MPO and DNA, measuring the typical components of NETs, DNA in combination with histones and neutrophil granule proteins. High correlations indicated that nucleosomes were, at the least, to a large extent derived from NET-forming neutrophils (supplementary figure S3).

Finally, our results suggest a bidirectional interaction of corticosteroids and NETs. While a 7-day treatment with prednisone was associated with a beneficial effect to achieve clinical stability, an overall reduction of length of hospital stay and a reduction in duration of i.v. antibiotic treatment [9], corticosteroids modulated NETosis, leading to a slower and more sustained decline of markers of NETs without increasing overall AUC after 3 days. Similarly, the subgroup of patients in the placebo group presenting with more sustained NETs levels from day 3 achieved clinical stability more rapidly than those with a faster decline. Accordingly, the temporal dynamics seem to exert a pivotal influence on clinical outcome: while excessive NETosis at baseline with consecutive exhaustion was associated with adverse outcome, moderate initial NET levels of sustained character were associated with a more beneficial outcome (supplementary figure S4). Against this background, beneficial effects of corticosteroids as adjunct therapy in CAP treatment may partly be driven by modulation of NET formation or pre-activation of neutrophils in the course of infection. In fact, significant effect 
modification of corticosteroid effects on TTCS by NET levels supports this assumption. Yet, known effects of glucocorticoids on neutrophils prolonging the survival, increasing the mobilisation from the bone marrow and reducing marginalisation in the vasculature could in part account for enhanced NETosis [42, 43]. As there are no data on the effects of corticosteroids on NETosis to date, the underlying pathophysiological mechanisms are yet to be determined.

In conclusion, our findings suggest that cell-free nucleosomes serve as a surrogate marker of NETosis, which appears to be a highly dynamic and prognostic cellular antimicrobial defence mechanism in CAP. The data contribute fundamentally to the understanding of the pathophysiology of lower respiratory tract infections, which are largely driven by neutrophil activation. While NET formation is understandably necessary in microbial defence, modulators of NETosis may have potential therapeutic benefits to limit excessive neutrophil recruitment and activation while preserving host defence.

The main strengths of our study include: 1) the well-characterised cohort of patients with CAP of different severities representative for patients usually treated in emergency departments and hospitals, 2) the large number of patients, 3) the assessment of clinically relevant end-points, and 4) the high accuracy and reproducibility of the measurement methods. However, limitations that need to be considered include: 1) this was a secondary analysis of a randomly selected subgroup, 2) measured cell-free nucleosomes may not only mirror NET formation but could partly reflect other mechanisms of cell death such as necrosis or necroptosis, 3) quantified markers of NETs could be overestimated due to possible fragmentation and 4) the results may not be applicable to patients with less severe CAP, who do not require hospitalisation.

Further studies are required to characterise NETosis in lower respiratory tract infections and to determine the relative importance to the progression and remission of the condition, and whether targeting this pathway would be of therapeutic benefit to patients' outcomes.

\section{Acknowledgements}

We thank the members of the STEP Study Team (names and affiliations listed in the supplementary material) for patient recruitment and data organisation.

Author contributions: B. Mueller, F. Ebrahimi, M. Christ-Crain, P. Hasler, P. Schuetz, S. Giaglis and S.V. van Breda conceived and designed the study, and organised and interpreted the data. S. Giaglis and S.V. van Breda performed the experiments. F. Ebrahimi, P. Hasler, P. Schuetz, S. Giaglis, A. Kutz, C.A. Blum, C. Baumgartner and S. Hahn drafted the manuscript for important intellectual content. All authors approved the final version to be published. F. Ebrahimi and P. Schuetz had full access to all of the data in the study, and take responsibility for the integrity of the data and the accuracy of the data analysis.

\section{References}

1 Thomas CP, Ryan M, Chapman JD, et al. Incidence and cost of pneumonia in Medicare beneficiaries. Chest 2012; 142: 973-981.

2 Shindo $\mathrm{Y}$, Ito R, Kobayashi $\mathrm{D}$, et al. Risk factors for 30-day mortality in patients with pneumonia who receive appropriate initial antibiotics: an observational cohort study. Lancet Infect Dis 2015; 15: 1055-1065.

3 Quan TP, Fawcett NJ, Wrightson JM, et al. Increasing burden of community-acquired pneumonia leading to hospitalisation, 1998-2014. Thorax 2016; 71: 535-542.

4 Zemans RL, Colgan SP, Downey GP. Transepithelial migration of neutrophils: mechanisms and implications for acute lung injury. Am J Respir Cell Mol Biol 2009; 40: 519-535.

5 Brinkmann V, Reichard U, Goosmann C, et al. Neutrophil extracellular traps kill bacteria. Science 2004; 303 : 1532-1535.

6 Brinkmann V, Zychlinsky A. Beneficial suicide: why neutrophils die to make NETs. Nat Rev Microbiol 2007; 5: 577-582.

7 Barnado A, Crofford LJ, Oates JC. At the bedside: neutrophil extracellular traps (NETs) as targets for biomarkers and therapies in autoimmune diseases. J Leukoc Biol 2016; 99: 265-278.

8 Grayson PC, Kaplan MJ. At the bench: neutrophil extracellular traps (NETs) highlight novel aspects of innate immune system involvement in autoimmune diseases. J Leukoc Biol 2016; 99: 253-264.

9 Blum CA, Nigro N, Briel M, et al. Adjunct prednisone therapy for patients with community-acquired pneumonia: a multicentre, double-blind, randomised, placebo-controlled trial. Lancet 2015; 385: 1511-1518.

10 Mandell LA, Wunderink RG, Anzueto A, et al. Infectious Diseases Society of America/American Thoracic Society consensus guidelines on the management of community-acquired pneumonia in adults. Clin Infect Dis 2007; 44: Suppl. 2, S27-S72.

11 Fine MJ, Auble TE, Yealy DM, et al. A prediction rule to identify low-risk patients with community-acquired pneumonia. N Engl J Med 1997; 336: 243-250.

12 Kessenbrock K, Krumbholz M, Schonermarck U, et al. Netting neutrophils in autoimmune small-vessel vasculitis. Nat Med 2009; 15: 623-625.

13 Sur Chowdhury C, Giaglis S, Walker UA, et al. Enhanced neutrophil extracellular trap generation in rheumatoid arthritis: analysis of underlying signal transduction pathways and potential diagnostic utility. Arthritis Res Ther 2014; 16: R122.

14 Diaz JA, Fuchs TA, Jackson TO, et al. Plasma DNA is elevated in patients with deep vein thrombosis. J Vasc Surg Venous Lymphat Disord 2013; 1: 341-348. 
15 Sur Chowdhury C, Hahn S, Hasler P, et al. Elevated levels of total cell-free DNA in maternal serum samples arise from the generation of neutrophil extracellular traps. Fetal Diagn Ther 2016; 40: 263-267.

16 Zhong X-Y, Muhlenen IV, Li Y, et al. Increased concentrations of antibody-bound circulatory cell-free DNA in rheumatoid arthritis. Clin Chem 2007; 53: 1609-1614.

17 Wang Y, Xiao Y, Zhong L, et al. Increased neutrophil elastase and proteinase 3 and augmented NETosis are closely associated with beta-cell autoimmunity in patients with type 1 diabetes. Diabetes 2014; 63: 4239-4248.

18 Carestia A, Frechtel G, Cerrone G, et al. NETosis before and after hyperglycemic control in type 2 diabetes mellitus patients. PLoS One 2016; 11: e0168647.

19 Fuchs TA, Alvarez JJ, Martinod K, et al. Neutrophils release extracellular DNA traps during storage of red blood cell units. Transfusion 2013; 53: 3210-3216.

20 Demers M, Krause DS, Schatzberg D, et al. Cancers predispose neutrophils to release extracellular DNA traps that contribute to cancer-associated thrombosis. Proc Natl Acad Sci USA 2012; 109: 13076-13081.

21 Douda DN, Jackson R, Grasemann $\mathrm{H}$, et al. Innate immune collectin surfactant protein D simultaneously binds both neutrophil extracellular traps and carbohydrate ligands and promotes bacterial trapping. J Immunol 2011; 187: $1856-1865$.

22 Narasaraju T, Yang E, Samy RP, et al. Excessive neutrophils and neutrophil extracellular traps contribute to acute lung injury of influenza pneumonitis. Am J Pathol 2011; 179: 199-210.

23 Bruns S, Kniemeyer O, Hasenberg M, et al. Production of extracellular traps against Aspergillus fumigatus in vitro and in infected lung tissue is dependent on invading neutrophils and influenced by hydrophobin RodA. PLoS Pathog 2010; 6: e1000873.

24 Fuchs TA, Abed U, Goosmann C, et al. Novel cell death program leads to neutrophil extracellular traps. J Cell Biol 2007; 176: 231-241.

25 Bozinovski S, Anthony D, Anderson GP, et al. Treating neutrophilic inflammation in COPD by targeting ALX/ FPR2 resolution pathways. Pharmacol Ther 2013; 140: 280-289.

26 Stockley JA, Walton GM, Lord JM, et al. Aberrant neutrophil functions in stable chronic obstructive pulmonary disease: the neutrophil as an immunotherapeutic target. Int Immunopharmacol 2013; 17: 1211-1217.

27 Dicker AJ, Crichton ML, Pumphrey EG, et al. Neutrophil extracellular traps are associated with disease severity and microbiota diversity in patients with chronic obstructive pulmonary disease. J Allergy Clin Immunol 2017; 141: 117-127.

28 Grommes J, Soehnlein O. Contribution of neutrophils to acute lung injury. Mol Med 2011; 17: 293-307.

29 Zemans RL, Matthay MA. What drives neutrophils to the alveoli in ARDS? Thorax 2017; 72: 1-3.

30 Oerschuk CM, Mizgerd JP, Kubo H, et al. Adhesion molecules and cellular biomechanical changes in acute lung injury: Giles F. Filley Lecture. Chest 1999; 116: 37S-43S.

31 Saffarzadeh M, Juenemann C, Queisser MA, et al. Neutrophil extracellular traps directly induce epithelial and endothelial cell death: a predominant role of histones. PLoS One 2012; 7: e32366.

32 Benites-Zapata VA, Hernandez AV, Nagarajan V, et al. Usefulness of neutrophil-to-lymphocyte ratio in risk stratification of patients with advanced heart failure. Am J Cardiol 2015; 115: 57-61.

33 Hosseinzadeh A, Thompson PR, Segal BH, et al. Nicotine induces neutrophil extracellular traps. J Leukoc Biol 2016; 100: 1105-1112.

34 Pedersen F, Marwitz S, Holz O, et al. Neutrophil extracellular trap formation and extracellular DNA in sputum of stable COPD patients. Respir Med 2015; 109: 1360-1362.

35 Lee J, Luria A, Rhodes C, et al. Nicotine drives neutrophil extracellular traps formation and accelerates collagen-induced arthritis. Rheumatology 2017; 56: 644-653.

36 Saukkonen K, Lakkisto P, Pettila V, et al. Cell-free plasma DNA as a predictor of outcome in severe sepsis and septic shock. Clin Chem 2008; 54: 1000-1007.

37 Dwivedi DJ, Toltl LJ, Swystun LL, et al. Prognostic utility and characterization of cell-free DNA in patients with severe sepsis. Crit Care 2012; 16: R151.

38 Yang S, Qi H, Kan K, et al. Neutrophil extracellular traps promote hypercoagulability in patients with sepsis. Shock 2017; 47: 132-139.

39 Zeerleder S, Stephan F, Emonts M, et al. Circulating nucleosomes and severity of illness in children suffering from meningococcal sepsis treated with protein C. Crit Care Med 2012; 40: 3224-3229.

40 Zeerleder S, Zwart B, Wuillemin WA, et al. Elevated nucleosome levels in systemic inflammation and sepsis. Crit Care Med 2003; 31: 1947-1951.

41 Moorthy AN, Rai P, Jiao H, et al. Capsules of virulent pneumococcal serotypes enhance formation of neutrophil extracellular traps during in vivo pathogenesis of pneumonia. Oncotarget 2016; 7: 19327-19340.

42 Liles WC, Dale DC, Klebanoff SJ. Glucocorticoids inhibit apoptosis of human neutrophils. Blood 1995; 86: 3181-3188.

43 Drewniak A, van Raam BJ, Geissler J, et al. Changes in gene expression of granulocytes during in vivo granulocyte colony-stimulating factor/dexamethasone mobilization for transfusion purposes. Blood 2009; 113: 5979-5998. 